\title{
The nature of the light variations of chemically peculiar stars CU Vir and HD 64740
}

\author{
Jiri Krtička ${ }^{1}$, Hana Marková ${ }^{1}, Z_{\text {Zdenek Mikulášek }}{ }^{1}$, \\ Theresa Lüftinger ${ }^{2}$, David Bohlender ${ }^{3}$, Juraj Zverko ${ }^{4}$, \\ and Jozef Žižňovský ${ }^{4}$ \\ ${ }^{1}$ Masaryk University, Kotlářská 2, CZ-61137 Brno, Czech Republic \\ email: krticka@physics.muni.cz, 175960@mail.muni.cz, mikulas@physics.muni.cz \\ ${ }^{2}$ Institute for Astronomy of the University of Vienna, Vienna, Austria \\ email: lueftinger@astro.univie.ac.at \\ ${ }^{3}$ National Research Council of Canada, Herzberg Institute of Astrophysics, Victoria, Canada \\ email: david.bohlender@nrc-cnrc.gc.ca \\ ${ }^{4}$ Astronomical Institute, Slovak Academy of Sciences, Tatranská Lomnica, Slovakia \\ email: zve@ta3.sk, ziga@ta3.sk
}

\begin{abstract}
The nature of the light variations of chemically peculiar stars was studied in detail only in a very few cases. To better understand the mechanisms of light variability of these stars, we study the light variations of the well-known magnetic chemically peculiar star CU Vir and one of the least amplitude variable stars HD 64740. We show that the light variability of these stars is induced by flux redistribution in spots of enhanced abundance of chemical elements (e.g., helium, silicon, iron or chromium), and by the stellar rotation. We conclude that this is a promising model for the explanation of the light variability of most chemically peculiar stars.
\end{abstract}

Keywords. stars: atmospheres, stars: chemically peculiar, stars: spots, stars: variables: others

\section{Modelling of light variations of magnetic chemically peculiar stars}

The light variations of magnetic chemically peculiar stars are still not very well understood. These stars show inhomogeneous surface distribution of different elements, including helium, silicon or iron. Krtička et al. (2007, 2009) showed that the redistribution of the emergent flux due to the bound-free (continuum) and bound-bound (line) transitions in the stellar atmosphere can explain the light variability of HD 37776, and HR 7224. They concluded that the comparison of observed and simulated light variations can provide an important new test of modern model stellar atmospheres.

The model stellar atmospheres are calculated using the code TLUSTY (Hubeny \& Lanz 1995, Lanz \& Hubeny 2007) assuming a fixed stellar effective temperature and surface gravity. The abundance of chemical elements concerned is set according to the maps of surface elemental distribution derived from spectroscopy.

The emergent fluxes from individual surface elements are calculated using the code SYNSPEC. From these fluxes the magnitudes of the star in individual photometric filters are derived. Finally, these simulated light variations are compared with the observed ones. We do not use any free parameter to fit the observed light curves.

\section{Results}

The observed light variations of CU Vir in vby filters of Strömgren photometric system can be explained as a result of inhomogeneous distribution of silicon, chromium, and 

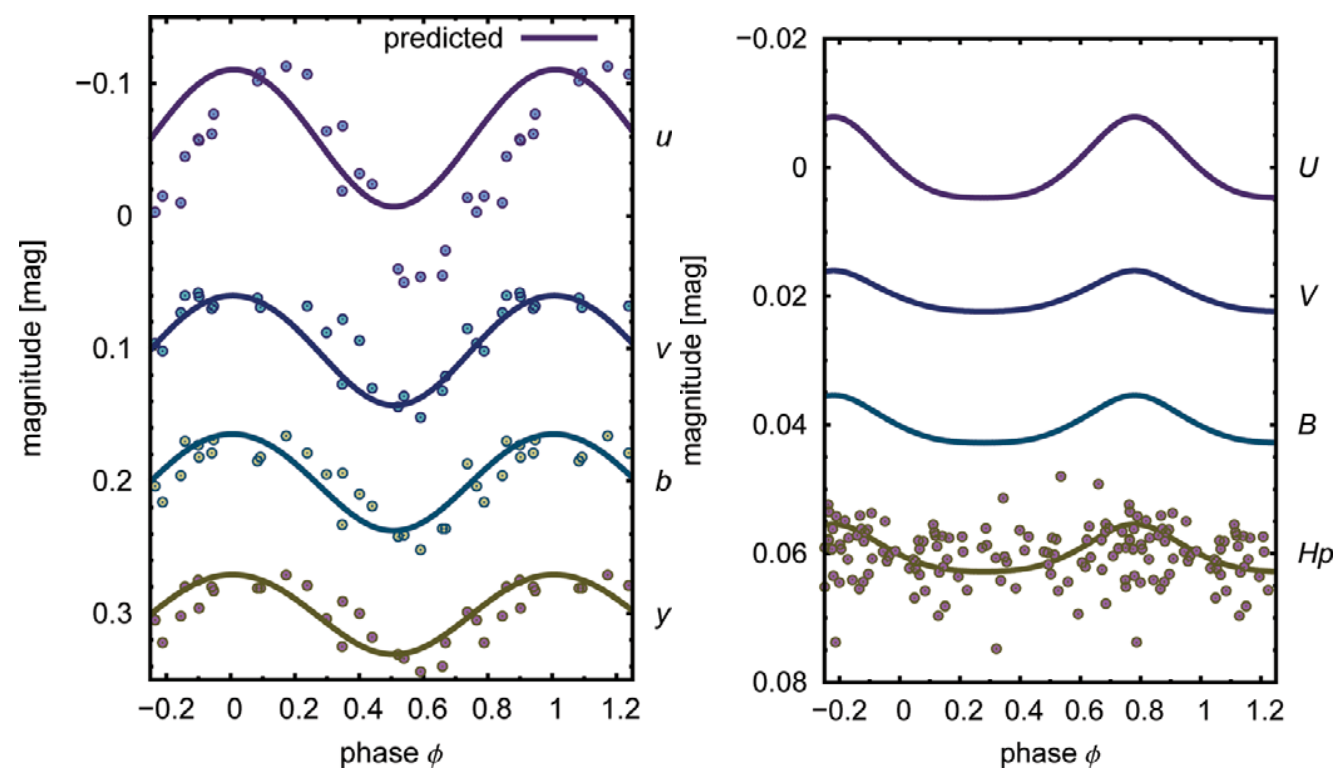

Figure 1. Left: Comparison of the predicted light variations of CU Vir (calculated using the helium, silicon, chromium, and iron surface distribution according to Kuschnig et al. (1999)) and the observed one (Pyper \& Adelman 1985) in different colors. Right: Comparison of the predicted light variations of HD 64740 (calculated using the helium and silicon surface distribution according to Bohlender \& Landstreet 1990), and the observed one (Perryman 1997).

iron on the stellar surface (see Fig. 1). This causes the redistribution of the flux from the ultraviolet to the visible part of the spectrum, and, due to the stellar rotation, the light variations. However, there is still some disagreement in the $u$ filter, which will be the subject of the further research.

The reason why some stars show only very small amplitude of the light variations is that their surface chemical composition does not significantly differ from the solar one. This was shown for one of the least amplitude variable stars, HD 64740. The low-amplitude light variations of this star observed by the Hipparcos satellite can be explained by the inhomogeneous surface distribution of helium and silicon (Fig. 1).

Comparison of the observed and simulated light variations serves as an independent test of modern model stellar atmospheres.

\section{Acknowledgements}

This work was supported by grants GAAV IAA301630901, MEB 060807/WTZ CZ11/2008, VEGA 2/0074/09, and MUNI/A/0968/2009.

\section{References}

Bohlender, D. A. \& Landstreet, J. D. 1990, ApJ, 358, 274

Hubeny, I. \& Lanz, T. 1995, ApJ, 439, 875

Kuschnig, R., Ryabchikova, T. A., Piskunov, N. E., \& Weiss, W. W. et al. 1999, A\&SA, 348, 924

Krtička, J., Mikuláček, Z., Zverko, J., \& Žižńovský, J. 2007, AESA, 470, 1089

Krtička, J., Mikulášek, Z., Henry, G. W., \& Zverko, J. et al. 2009, A\&\&A, 499, 567

Lanz, T. \& Hubeny, I. 2007, ApJS, 169, 83

Perryman M.A.C. \& ESA 1997, The Hipparcos and Tycho Catalogues, ESA-SP 1200 (Noordwijk, Netherlands: ESA Publications Division)

Pyper, D. M. \& Adelman, S. J. 1985, A\& AS, 59, 369 